Article

\title{
Reduced Sulfation Enhanced Oxytosis and Ferroptosis in Mouse Hippocampal HT22 Cells
}

\author{
Haruna Nagase ${ }^{1}$, Yasuhiro Katagiri ${ }^{2}$, Kentaro Oh-hashi ${ }^{1,3}$, Herbert M. Geller ${ }^{2}$ and \\ Yoko Hirata 1,3,*(D) \\ 1 United Graduate School of Drug Discovery and Medical Information Sciences, Gifu University, Yanagido, \\ Gifu 501-1193, Japan; haruna.science@gmail.com (H.N.); oohashi@gifu-u.ac.jp (K.O.-h.) \\ 2 Laboratory of Developmental Neurobiology, National Heart, Lung, and Blood Institute, NIH, \\ Bethesda, MD 20892, USA; katagiry@nhlbi.nih.gov (Y.K.); gellerh@nhlbi.nih.gov (H.M.G.) \\ 3 Department of Chemistry and Biomolecular Science, Faculty of Engineering, Gifu University, Yanagido, \\ Gifu 501-1193, Japan \\ * Correspondence: yokoh@gifu-u.ac.jp; Tel.: +81-58-293-2609
}

Received: 8 December 2019; Accepted: 4 January 2020; Published: 6 January 2020

\begin{abstract}
Sulfation is a common modification of extracellular glycans, tyrosine residues on proteins, and steroid hormones, and is important in a wide variety of signaling pathways. We investigated the role of sulfation on endogenous oxidative stress, such as glutamate-induced oxytosis and erastin-induced ferroptosis, using mouse hippocampal HT22 cells. Sodium chlorate competitively inhibits the formation of $3^{\prime}$-phosphoadenosine $5^{\prime}$-phosphosulfate, the high energy sulfate donor in cellular sulfation reactions. The treatment of HT22 cells with sodium chlorate decreased sulfation of heparan sulfate proteoglycans and chondroitin sulfate proteoglycans. Sodium chlorate and $\beta$-D-xyloside, which prevents proteoglycan glycosaminoglycan chain attachment, exacerbated both glutamate- and erastin-induced cell death, suggesting that extracellular matrix influenced oxytosis and ferroptosis. Moreover, sodium chlorate enhanced the generation of reactive oxygen species and influx of extracellular $\mathrm{Ca}^{2+}$ in the process of oxytosis and ferroptosis. Interestingly, sodium chlorate did not affect antioxidant glutathione levels. Western blot analysis revealed that sodium chlorate enhanced erastin-induced c-Jun N-terminal kinase phosphorylation, which is preferentially activated by cell stress-inducing signals. Collectively, our findings indicate that sulfation is an important modification for neuroprotection against oxytosis and ferroptosis in neuronal hippocampal cells.
\end{abstract}

Keywords: oxidative stress; oxytosis; ferroptosis; proteoglycans; sodium chlorate; sulfation

\section{Introduction}

Alzheimer's disease and Parkinson's disease are the two most common neurodegenerative diseases to have become increasingly prevalent, especially in the grayer society. These diseases are characterized by a progressive neuronal loss in which many factors are involved in neuronal cell death, including oxidative stress. Cellular models of endogenous oxidative stress such as oxytosis and ferroptosis are an iron-dependent form of non-apoptotic cell death, and reproduce many of the abnormal characteristics in these neurodegenerative diseases [1]. It has been found that excessive extracellular glutamate or erastine inhibits the cystine/glutamate antiporter and depletes intracellular cysteine, leading to a reduction in glutathione (GSH) content and the accumulation of reactive oxygen species (ROS), which eventually cause oxidative stress-induced cell death or oxytosis/ferroptosis in HT22 cells [2-5]. HT22 is an immortalized mouse hippocampal cell line which is widely used as in vitro model for studying endogenous oxidative stress. Glutamate induces not only oxidative stress-induced cell death but also excitotoxic cell death through ionotropic glutamate receptors in neurons. Because 
HT22 cells lack functional ionotropic glutamate receptors, excitotoxicity can be excluded as a cause for glutamate-triggered cell death [2].

Previous reports have demonstrated that various natural and synthetic compounds against ROS-mediated damage prevented glutamate-induced endogenous oxidative stress [6-8]. These mainly target generation and accumulation of ROS within cells. On the other hand, many findings evidenced antioxidant properties of sulfated glycosaminoglycans (GAGs), particularly for heparan sulfate (HS) and chondroitin sulfate (CS) $[9,10]$, which may reduce oxidative damage extracellularly [11,12]. The majority of GAGs linked to protein to form proteoglycans such as heparan sulfate-proteoglycans (HSPGs) and chondroitin sulfate-proteoglycans (CSPGs) are located primarily on the cell surface or in the extracellular matrix (ECM) that lead to highly negatively charged structures. This cellular microenvironment might potentially be able to scavenge and bind cations, which may provide some neuroprotection against oxidative stress [13].

In this paper, we aimed to evaluate the effect of reduced sulfation on endogenous oxidative stress-induced cell death in mouse hippocampal HT22 cells using sodium chlorate, a widely used inhibitor of sulfation $[14,15]$, and $\beta$-D-xyloside, which prevents the attachment of sulfated GAG chains to core proteins [16]. The treatment of cells with sodium chlorate in order to reduce the sulfation of proteoglycans is suggested to be a useful method for examining possible functions of the sulfate groups $[14,15,17]$, while changes with xylosides implicate GAG chains in a process. We found that both treatments enhanced oxidative stress.

\section{Materials and Methods}

\subsection{Materials}

Erastin (Sigma-Aldrich Corporation, St. Louis, MO, USA, Cat\# E7781) and 4-methylumbelliferyl- $\beta$ D-xylopyranoside (Sigma-Aldrich Corporation, St. Louis, MO, USA, Cat\# M7008) were dissolved in cell culture-grade dimethyl sulfoxide and stored in the dark at $-20^{\circ} \mathrm{C}$. Phthaldialdehyde (Sigma-Aldrich Corporation, St. Louis, MO, USA, Cat\# P0657) was dissolved in methanol and freshly prepared. Sodium chlorate (FUJIFILIM Wako Pure Chemical, Osaka, Japan, Cat\# 193-01642) was dissolved in Dulbecco's modified Eagle's medium (DMEM; FUJIFILM Wako Pure Chemicals, Osaka, Japan, Cat\# 041-29775) and freshly prepared.

\subsection{Cell Culture}

HT22 immortalized hippocampal cells (RRID:CVCL_0321) were kindly provided by Dr. Schubert (The Salk Institute, La Jolla, CA, USA). The HT22 cells were cultured in DMEM (FUJIFILM Wako Pure Chemicals, Osaka, Japan) supplemented with $5 \%$ fetal bovine serum (HyClone Laboratories, Logan, UT, USA, Cat\# SH30406.02) at $37^{\circ} \mathrm{C}$ in a $5 \% \mathrm{CO}_{2}$ incubator.

\subsection{Immunofluorescent Staining and Imaging}

To evaluate HS and CS GAG chains, HT22 cells were stained with either monoclonal anti-HS antibody 10E4 (F58-10E4 clone, 1:250 dilution; AMS Biotechnology Ltd. Abingdon, UK, RRID:AB_10891554) or monoclonal anti-CS antibody CS-56 (anti-6S, 2S, 4S antibody) (1:100 dilution; Sigma-Aldrich Corporation, St. Louis, MO, USA, RRID:AB_476879). HT22 cells $\left(2.0 \times 10^{3} / \mathrm{cm}^{2}\right)$ were grown on 24 -well plate and treated with sodium chlorate. The cells were fixed with $4 \%$ paraformaldehyde for $15 \mathrm{~min}$ and then non-specific binding was blocked with $2.5 \%$ bovine serum albumin (BSA) in phosphate-buffered saline (PBS). Subsequently, HT22 cells were incubated in primary antibodies with $1 \%$ BSA or immunoenhancing reagent (Can Get Signal ${ }^{\circledR}$ Immunostain Solution B; TOYOBO, Osaka, Japan, Cat\# NKB-401) overnight at $4{ }^{\circ} \mathrm{C}$. HT22 cells were washed with PBS and immersed in $2.5 \%$ BSA or Can Get Signal ${ }^{\circledR}$ Immunostain Solution B (TOYOBO, Osaka, Japan) containing anti-IgM-FITC (fluorescein isothiocyanate, 1:250 dilution; Jackson ImmunoResearch, PA, USA) and then incubated with $10 \mu \mathrm{g} / \mathrm{mL}$ Hoechst 33258 (Molecular Probes, OR, USA) for nuclei staining. After 
washing with PBS, fluorescence images were captured with a fluorescence digital microscope (BZ-X800, Keyence, Osaka, Japan). The fluorescent intensity was quantified using Keyence image measurement and the analyzing software (BZ-X800 Analyzer, Keyence Corporation, Osaka, Japan).

\subsection{Western Blotting}

HT22 cells were lysed with sodium dodecyl sulfate (SDS) sample buffer (62.5 mM Tris- $\mathrm{HCl}$ (pH 6.8), $2 \%$ SDS, $10 \%$ glycerol, $0.1 \%$ bromophenol blue). Protein concentration in lysate was determined by the DC Protein Assay Kit (Bio-Rad Laboratories, Hercules, CA, USA, Cat\# 5000111JA) using $\gamma$-globulins as a standard. Equal amounts of protein $(20 \mu \mathrm{g})$ were separated by SDS-PAGE under reducing conditions and transferred to nitrocellulose membranes. Membranes were blocked with 5\% skim milk in PBS containing $0.05 \%$ Tween-20 (PBS-T) for $1 \mathrm{~h}$ at room temperature. Membranes were incubated with the following primary antibodies at the indicated dilutions: anti-phospho-SAPK/JNK (Thr183/Tyr185) (81E11) (1:1000 dilution, rabbit monoclonal, Cell Signaling Technology, Inc., Beverly, MA, USA, RRID:AB_823588), anti-HO-1 (1:2000 dilution, mouse monoclonal, Enzo Life Science, Inc., Farmingdale, NY, USA, RRID:AB_10617276), and anti-glyceraldehyde 3 phosphate dehydrogenase (GAPDH; 1:5000 dilution; mouse monoclonal, Acris Antibodies, Inc., San Diego, CA, USA, RRID:AB_1616730). Membranes were washed and incubated with an anti-mouse or anti-rabbit IgG secondary antibody conjugated to horseradish peroxidase (1:2000 dilution, Cell Signaling Technology, Inc.) in blocking buffer. Signals were visualized using Amersham ECL Western Blotting Detection Reagent (GE Healthcare UK Ltd., Buckinghamshire, England, Cat\# RPN2106) or SuperSignal ${ }^{\mathrm{TM}}$ West Dura Extended Substrate (Thermo Fisher Scientific, Waltham, MA, USA, Cat\# 34075) and Amersham Hyperfilm ECL (GE Healthcare UK Ltd., Buckinghamshire, UK, Cat\# 28906837). The blots were reprobed with different antibodies after stripping in $62.5 \mathrm{mM}$ Tris- $\mathrm{HCl}$ (pH 6.7), $100 \mathrm{mM} \beta$-mercaptoethanol, and $2 \%$ SDS at $50{ }^{\circ} \mathrm{C}$ for $30 \mathrm{~min}$, when required. Quantification of the bands was performed using ImageJ.

\subsection{Cell Viability Assay}

Cell viability was determined with 2-(4-Iodophenyl)-3-(4-nitrophenyl)-5-(2,4-disulfophenyl)-2Htetrazolium (WST-1) using a Cell counting Kit (Dojindo Laboratories, Kumamoto, Japan, Cat\# CK01). HT22 cells were cultured on 96-well plates and treated with sodium chlorate for the indicated period. Cultured medium was replaced to the medium containing $500 \mathrm{nM}$ WST-1 and incubated for additional $2 \mathrm{~h}$ at $37^{\circ} \mathrm{C}$ in a $5 \% \mathrm{CO}_{2}$ incubator. Absorbance was measured at $450 \mathrm{~nm}$ with $690 \mathrm{~nm}$ as a reference wavelength.

\subsection{Cell Death Assay}

Cytotoxicity was determined by measuring lactate dehydrogenase (LDH) released into the culture medium with a Cytotoxicity Detection Kit according to the manufacture's protocol (Takara Bio Inc., Shiga, Japan, Cat\# MK401). HT22 cells were grown on 48-well plates in $400 \mu \mathrm{L}$ of the medium. After the treatment of the cells under various experimental conditions, $10 \mu \mathrm{L}$ of the culture medium was analyzed for LDH activity. The percent cell death was calculated as $100 \times$ (experimental release blank)/(total release - blank), where total release is the activity from $10 \mu \mathrm{L}$ of cells lysed with $1 \%$ Triton X-100.

\subsection{GSH Determination}

HT22 cells were cultured on 6-well plates and treated under various experimental conditions. The cells were collected with cold PBS and centrifuged. The cell pellet was resuspended in $120 \mu \mathrm{L}$ of $0.1 \mathrm{M}$ sodium phosphate buffer ( $\mathrm{pH}$ 8.0)/5 mM EDTA, and deproteinized by adding four volumes of $25 \%(w / v)$ metaphosphoric acid. The sample was centrifuged for $10 \mathrm{~min}$ at $15,000 \times g$. The supernatant was used for GSH assay developed by Hissin and Hilf [18] with slight modification. Briefly, the supernatant $(5 \mu \mathrm{L}), 185 \mu \mathrm{L}$ of $0.1 \mathrm{M}$ sodium phosphate buffer ( $\mathrm{pH} 8.0$ ) containing $5 \mathrm{mM}$ EDTA and $10 \mu \mathrm{L}$ of phthaldialdehyde solution $(1 \mathrm{mg} / \mathrm{mL}$ in methanol) were added to a 96 -well black plate and 
incubated at $25{ }^{\circ} \mathrm{C}$ for $15 \mathrm{~min}$. Fluorescence intensity was measured at $420 \mathrm{~nm}$ with excitation at $350 \mathrm{~nm}$ using a microplate reader (Varioskan Flash; Tharmo Fischer Scientific, Waltham, MA, USA). The resultant pellet was solubilized in $75 \mu \mathrm{L} 0.2 \mathrm{M} \mathrm{NaOH}$ and used for protein assay. GSH was normalized to cellular protein measured by the DC Protein Assay (Bio-Rad Laboratories, Hercules, CA, USA).

\subsection{ROS Production}

HT22 cells were cultured on a 12-well plate and treated under various experimental conditions. MitoSOX (5 $\mu \mathrm{M}$; Thermo Fisher Scientific, Waltham, MA, USA, Cat\# M36008) was added and incubated for $15 \mathrm{~min}$ at $37^{\circ} \mathrm{C}$. The medium was replaced by serum-free DMEM without phenol red (Thermo Fisher Scientific, Waltham, MA, USA, Cat\# 21063029). The fluorescence was measured by a fluorescence digital microscope (BZ-X800, Keyence Corporation, Osaka, Japan). Fluorescent intensity was quantified using Keyence image measurement and the analyzing software (BZ-X800 Analyzer, Keyence Corporation, Osaka, Japan).

\section{9. $\mathrm{Ca}^{2+} \operatorname{Influx}$}

HT22 cells were grown on a 12-well plate and treated under various experimental conditions. Fluo4-AM (2 $\mu$ M; Thermo Fisher Scienctific, Waltham, MA, USA, Cat\# F14201) and Pluronic F-127 (0.04\%; Thermo Fisher Scientific, Waltham, MA, USA, Cat\# P3000MP) were added and incubated for $15 \mathrm{~min}$ at $37^{\circ} \mathrm{C}$. The medium was replaced by serum-free DMEM without phenol red. The fluorescence was measured by a fluorescence digital microscope (BZ-X800, Keyence Corporation). The fluorescent intensity was quantified using Keyence image measurement and the analyzing software (BZ-X800 Analyzer, Keyence Corporation, Osaka, Japan).

\subsection{Statistical Analyses}

The numerical data were statistically analyzed with GraphPad Prism 6.0 (GraphPad Software, Inc., La Jolla, CA, USA, PRID:SCR_002798). The significant differences between the control group and experimental group were determined by Bonferroni's multiple comparison tests following ANOVA.

\section{Results}

\subsection{Effect of Sodium Chlorate on Cell Viability in HT22 Cells}

We first examined the cytotoxicity of sodium chlorate in HT22 cell (Figure 1). Cell viability was not changed up to $40 \mathrm{mM}$ and decreased significantly at $60 \mathrm{mM}$ after the incubation of the cells for $24 \mathrm{~h}$ and $48 \mathrm{~h}$. Accordingly, we chose non-toxic $(20 \mathrm{mM})$ and slightly toxic $(60 \mathrm{mM})$ concentrations of sodium chlorate for further experiments.

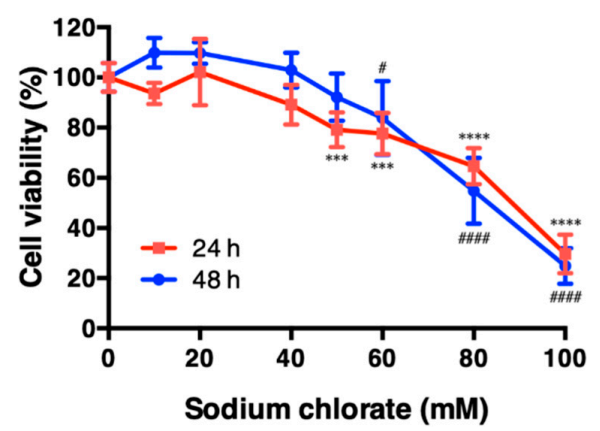

Figure 1. Effect of sodium chlorate on cell viability. HT22 cells were treated with the indicated concentrations of sodium chlorate for $24 \mathrm{~h}$ (red line) or $48 \mathrm{~h}$ (blue line) and cell viability was measured by WST-1 assay. The data are presented as the means \pm SD of at least six independent cultures. ${ }^{* * *} p<0.001,{ }^{* * * *} p<0.0001$ compared with the 24 h control; ${ }^{\#} p<0.05,{ }^{\# \# \#} p<0.0001$ compared with the 48-h control. 


\subsection{Sodium Chlorate Reduced the Sulfation of GAGs}

Proteoglycan GAG chains are highly sulfated. To check the efficacy of sodium chlorate treatment, we investigated the effect of sodium chlorate on the sulfation of HS and CS GAG chains in HT22 cells (Figure 2). HS was detected by the 10E4 antibody (F58-10E4 clone) which recognize common epitopes on HS including an N-sulfated glucosamine residue [19]. CS was detected by the CS-56 antibody which is specific for the GAG portion of native CSPGs. Treatment with $20 \mathrm{mM}$ sodium chlorate decreased both HS and CS, suggesting that sodium chlorate reduced sulfation of HSPGs and CSPGs (Figure 2a,b).

(a)
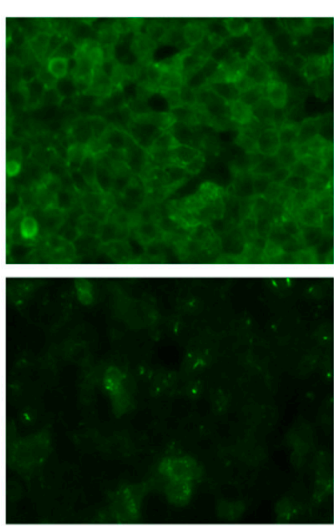

$\stackrel{\mathrm{sc}}{(20 \mathrm{mM})}$

$\mathrm{SC}$

(60 mM)

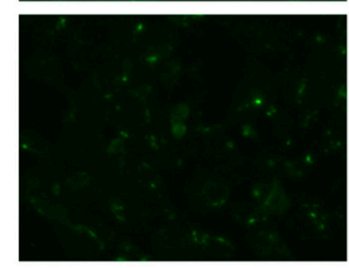

Hoechst 33258
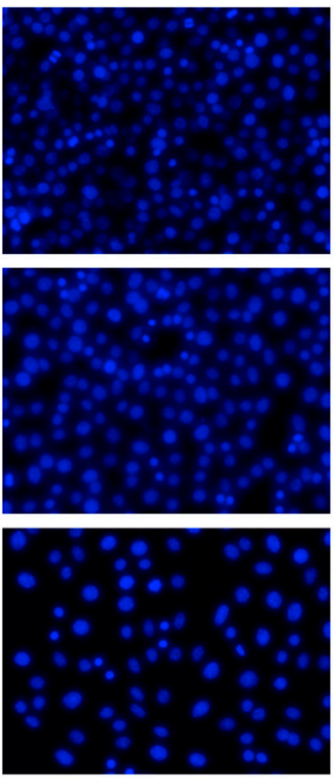

CS
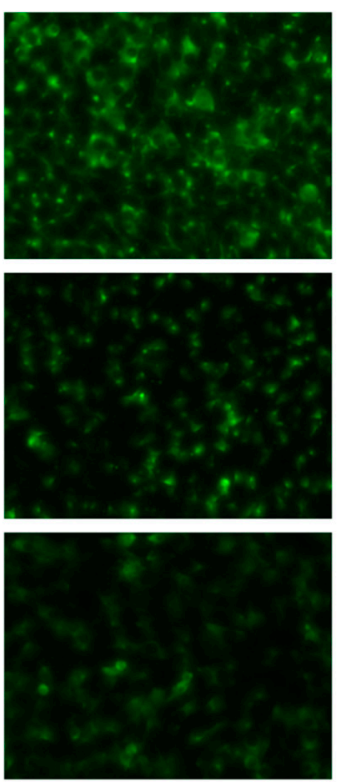

Hoechst 33258
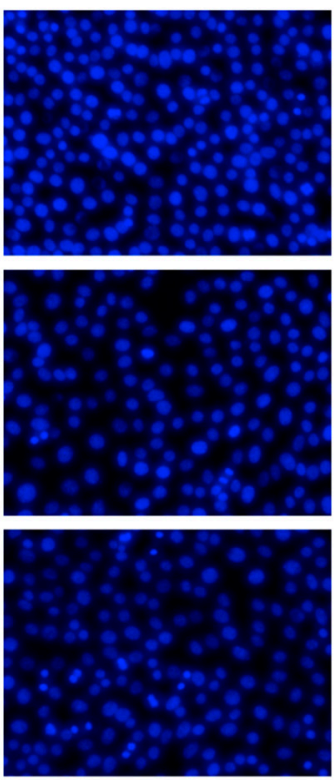

(b)
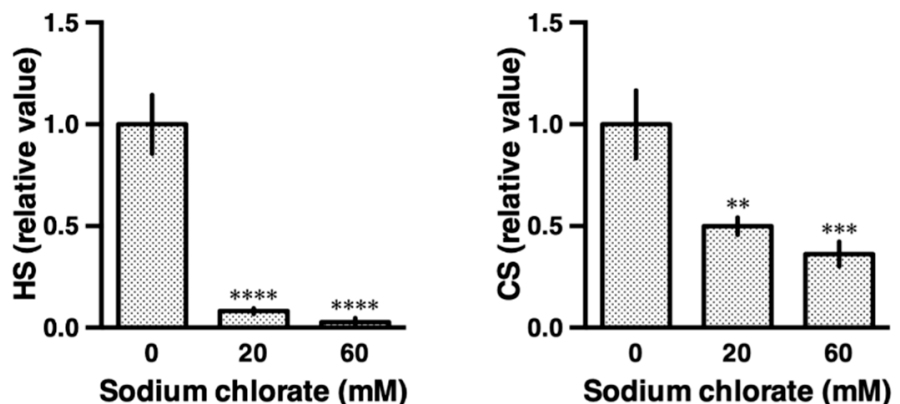

Figure 2. Effect of sodium chlorate on HS and CS in HT22 cells. (a) Representative fluorescence micrographs of typical data shown. HT22 cells were cultured with or without sodium chlorate (20 or $60 \mathrm{mM}$ ) for $24 \mathrm{~h}$ and subjected to immunofluorescent staining. HT22 cells were stained with 10E4 (anti-HS antibody) and CS-56 (anti-CS antibody). (b) Fluorescence intensity was quantified using a Keyence image measurement and analyzing software (VH-H1A5; Keyence). The data are presented as the mean $\pm \operatorname{SD}(n=4) .{ }^{* *} p<0.01,{ }^{* * *} p<0.001,{ }^{* * * *} p<0.0001$ compared with the control.

\subsection{Sodium Chlorate Treatment Enhanced Extracellular Glutamate-and Erastin-Induced Cell Death in HT22 Cells}

Next, we examined the effect of sodium chlorate on glutamate- and erastin-induced oxidative stress. Because $10 \mathrm{mM}$ glutamate and 0.5-1 $\mu \mathrm{M}$ erastin caused nearly maximal cell death (data not shown), we chose $5 \mathrm{mM}$ and $0.2 \mu \mathrm{M}$, respectively, as submaximal concentrations. Sodium chlorate enhanced both glutamate- and erastin-induced cell death in a concentration-dependent manner (Figure 3). Although cell death by LDH assay in Figure 3 showed no increase up to $60 \mathrm{mM}$ sodium 
chlorate, total LDH activity which is proportional to cell number decreased (data not shown), indicating that cell proliferation was suppressed under this condition. The data are consistent with the result obtained from cell viability by WST-1 assay shown in Figure 1. These results suggest that reduced sulfation exacerbated both glutamate-induced oxytosis and erastin-induced ferroptosis. Because erastin-induced cell death was more affected by sodium chlorate than glutamate-induced cell death, we focused on erastin-induced cell death, ferroptosis, for further experiments.

(a)

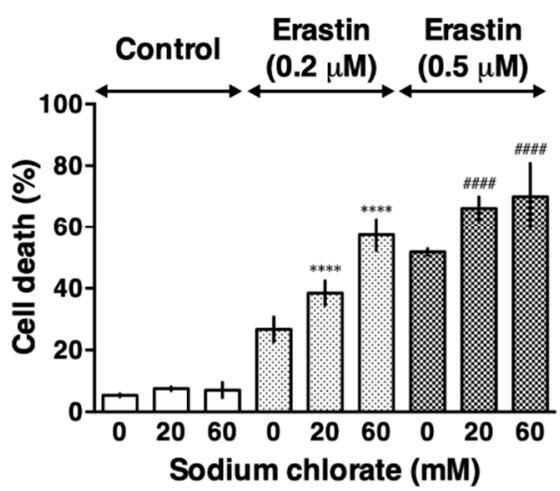

(b)

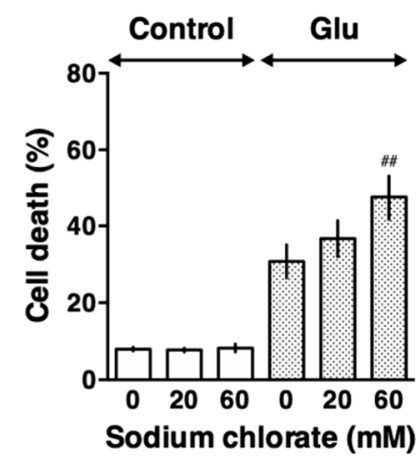

Figure 3. Sodium chlorate enhanced endogenous oxidative stress-induced cell death in HT22 cells. (a) Effect of sodium chlorate on erastin-induced cell death. HT22 cells were treated with $0.2 \mu \mathrm{M}$ or $0.5 \mu \mathrm{M}$ erastin in the presence or absence of sodium chlorate $(20$ or $60 \mathrm{mM})$ for $24 \mathrm{~h}$ and cell death was determined by LDH assay. (b) Effect of sodium chlorate on glutamate-induced cell death. HT22 cells were treated with $5 \mathrm{mM}$ glutamate in the presence or absence of sodium chlorate (20 or $60 \mathrm{mM})$ for $24 \mathrm{~h}$ and cell death was determined by LDH assay. The data are presented as the mean \pm SD. The data were obtained from at least four independent cultures. ${ }^{* * *} p<0.0001,{ }^{\# \# \#} p<0.0001$ compared with $0.2 \mu \mathrm{M}$ or $0.5 \mu \mathrm{M}$ erastin alone; ${ }^{\# \#} p<0.01$ compared with glutamate alone.

\section{4. $\beta$-D-Xyloside Enhanced Extracellular Glutamate-and Erastin-Induced Cell Death in HT22 Cells}

Although sodium chlorate reduced HS and CS efficiently in HT22 cells, it potentially inhibits sulfation of not only extracellular glycans but also tyrosine residues on proteins and steroid hormones, which is important in many signaling pathways. Therefore, we used 4-methylumbelliferyl- $\beta$-D-xylopyranoside ( $\beta$-D-xyloside), a compound which interferes with proteoglycan synthesis by acting as an artificial acceptor for glycosaminoglycan synthesis and thereby competing with the proteoglycan core protein [20]. The treatment of the cells with $\beta$-D-xyloside also caused a reduction in HS and CS immunoreactivity as well as exacerbation of erastin-induced ferroptoosis (Figure 4a,b). These findings confirmed that the reduced synthesis of HS and CS enhanced endogenous oxidative stress-induced cell death.

\subsection{Sodium Chlorate Treatment Enhanced Erastin-Induced ROS Production but Did Not Affect GSH Depletion in HT22 Cells}

Earlier studies have demonstrated that the glutamate- and erastin-induced oxidative stress is initiated by inhibiting the transmembrane cystine/glutamate antiporter, leading to depletion of intracellular GSH, accumulation of ROS, and excess influx of $\mathrm{Ca}^{2+}$, resulting in cell death in HT22 cells [2-5]. To investigate whether reduced sulfation also affects to the cell death cascade, intracellular GSH, accumulation of ROS and an influx of $\mathrm{Ca}^{2+}$ were examined after the treatment with erastin in the presence or absence of sodium chlorate. As reported previously, erastin increased the production of ROS which was enhanced by treatment with sodium chlorate (Figure 5b). Similarly, an erastin-induced influx of $\mathrm{Ca}^{2+}$ was accelerated by sodium chlorate (Figure 5c). These results suggested that reduced sulfation is accompanied by increased ROS production and $\mathrm{Ca}^{2+}$ influx, leading to the exacerbation of ferroptotic cell death. In contrast, GSH levels decreased similarly in erastin-treated HT22 cells in the 
presence or absence of sodium chlorate (Figure 5a). These results indicate that reduced sulfation did not affect cystine-glutamate transport via system xc-, a cystine/glutamate antiporter.

(a)

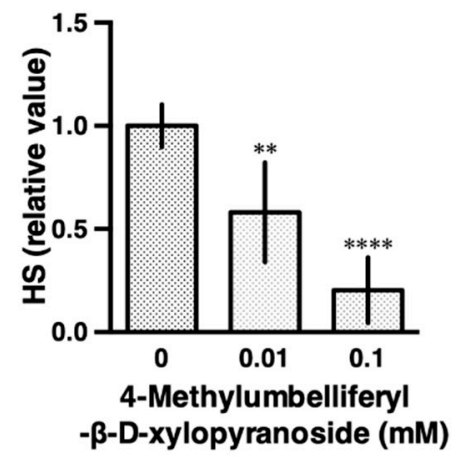

(b)

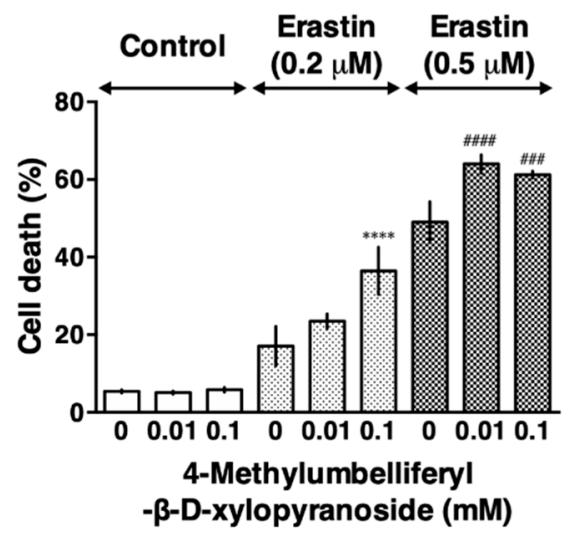

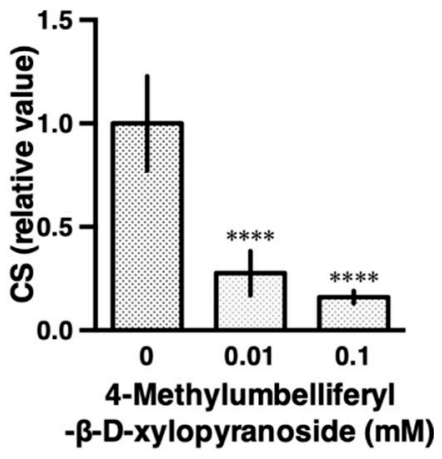

- $\beta$-D-xylopyranoside (mM)

Figure 4. Effect of $\beta$-D-xyloside on HS and CS levels and erastin-induced cell death. To examine the effect of $\beta$-D-xyloside, HT22 cells were cultured with the indicated concentrations of 4-methylumbelliferyl- $\beta$-D-xylopyranoside for 2 days. The cells were plated on 24 -well plates for immunofluorescent staining of HS and CS or 48 well plates for cell death assay. (a) HT22 cells were cultured for another $24 \mathrm{~h}$ in the presence of 4-methylumbelliferyl- $\beta$-D-xylopyranoside and stained with 10E4 (anti-HS antibody) and CS-56 (anti-CS antibody), respectively. Fluorescence intensity was quantified using a Keyence image measurement and analyzing software (VH-H1A5; Keyence). The data are presented as the mean $\pm \mathrm{SD}(n=7-9)$. ${ }^{* *} p<0.01,{ }^{* * *} p<0.0001$ compared with the control. (b) HT22 cells were treated with $0.2 \mu \mathrm{M}$ or $0.5 \mu \mathrm{M}$ erastin in the presence or absence of 4-methylumbelliferyl- $\beta$-D-xylopyranoside $(0.01$ or $0.1 \mathrm{mM})$ for $24 \mathrm{~h}$ and cell death was determined by LDH assay. The data are presented as the mean \pm SD. The data were obtained from at least four independent cultures. ${ }^{* * * *} p<0.0001$ compared with $0.2 \mu \mathrm{M}$ erastin alone; ${ }^{\# \# \#} p<0.001$, \#\#\#\# $p<0.0001$ compared with $0.5 \mu \mathrm{M}$ erastin alone.

\subsection{Effect of ROS Scavenging and Iron Chelating Chemicals on Sodium Chlorate-Enhanced Ferroptotic Cell Death}

Several studies have reported that ROS scavenging and iron chelating chemicals such as $\alpha$-tocopherol and deferoxamine prevent glutamate- or erastin-induced cell death in HT22 cells [21-23]. We examined whether ROS scavenging and iron chelating chemicals mitigated enhanced toxicity of sodium chlorate. Cotreatment with $\alpha$-tocopherol $(1 \mu \mathrm{M})$ completely suppressed erastin-induced cell death irrespective of the presence or absence of sodium chlorate. In contrast, the protective effect of $\alpha$-tocopherol $(0.1 \mu \mathrm{M}$ or less) weakened significantly in the presence of sodium chlorate (Figure $6 \mathrm{a})$. Similarly, deferoxamine ( $5 \mu \mathrm{M}$ or more) completely suppressed erastin-induced cell death, however, deferoxamine $(2 \mu \mathrm{M})$ only partially prevented enhanced toxicity of sodium chlorate (Figure $6 \mathrm{~b}$ ). These results indicate that increased $\mathrm{ROS}$ and $\mathrm{Fe}^{2+}$ are involved in sodium chlorate-enhanced ferroptotic cell death. 
(a)

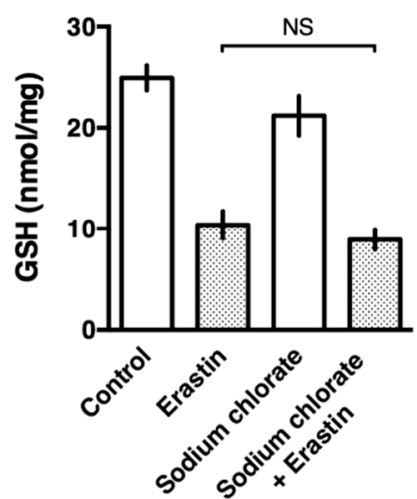

(b)

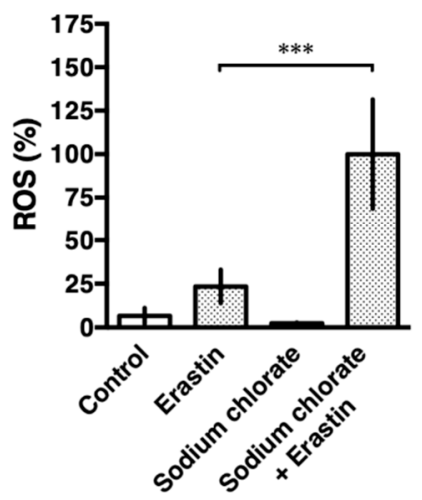

(c)

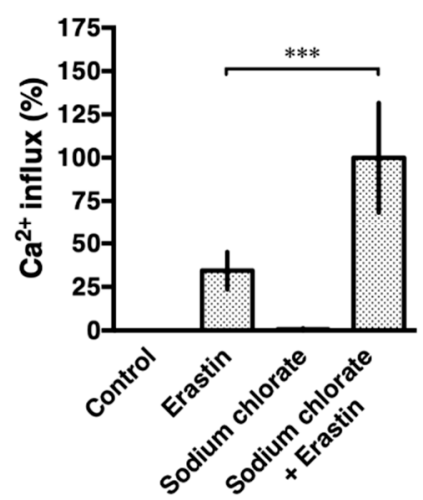

Figure 5. Effects of sodium chlorate on GSH contents, ROS production, and $\mathrm{Ca}^{2+}$ influx in erastin-treated HT22 cells. The cells were treated with $0.2 \mu \mathrm{M}$ erastin in the presence or absence of $20 \mathrm{mM}$ sodium chlorate for $8 \mathrm{~h}$. (a) Glutathione contents were measured and normalized to the protein concentration. (b) ROS production was determined with MitoSOX reagent. (c) Intracellular $\mathrm{Ca}^{2+}$ was detected with Fluo- 4 reagent. The data are presented as the mean $\pm \mathrm{SD}$. The data were obtained from at least three independent cultures. ${ }^{* * *} p<0.001$; NS, not significant.

(a)

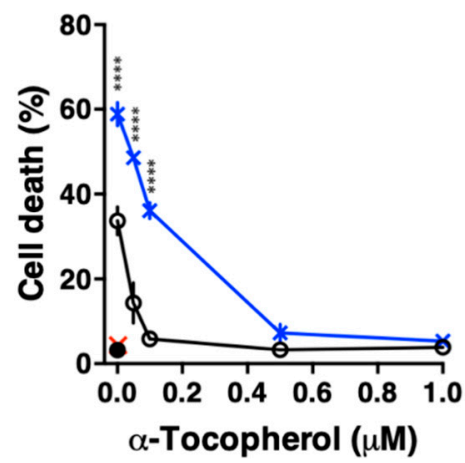

(b)

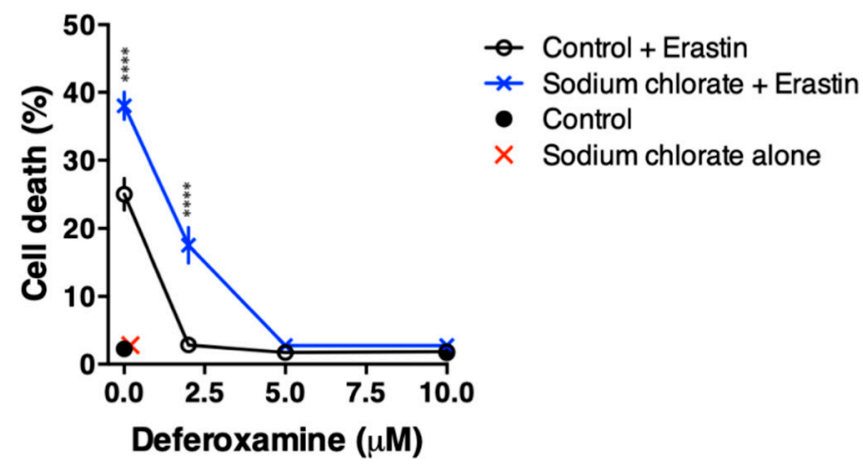

Figure 6. Effect of $\alpha$-tocopherol or deferoxamine on erastin-induced cell death in the presence or absence of sodium chlorate. (a) ROS scavenging by $\alpha$-tocopherol mitigated enhanced toxicity of sodium chlorate. (b) Iron chelating by deferoxamine mitigated enhanced toxicity of sodium chlorate. HT22 cells were treated with $0.2 \mu \mathrm{M}$ erastin and indicated concentrations of $\alpha$-tocopherol or deferoxamine in the presence or absence of $60 \mathrm{mM}$ sodium chlorate for $24 \mathrm{~h}$ and cell death was determined by LDH assay. The data are presented as the mean $\pm \mathrm{SD}$. The data were obtained from at least four independent cultures. ${ }^{* * *} p<0.0001$ compared with control + erastin at the same concentration.

3.7. Sodium Chlorate Treatment Enhanced Erastin-Induced Phosphorylation of c-Jun N-Terminal Kinase (JNK) in HT22 Cells

Oxidative stress directly or indirectly contributes to ROS-mediated various intracellular signaling pathway. ROS activates the Nrf2-antioxidant response element (ARE) pathway, an indicator and modulator of oxidative stress in neurodegeneration [24] that induces the expression of downstream target genes, such as hemoxygenase-1 (HO-1). ROS also induces phosphorylation of the stress kinase JNK which is involved in cellular responses to environmental stresses. We investigated whether sodium chlorate affected these intracellular signaling pathways in response to oxidative stress. Consistent with the previous reports [25], phosphorylation of JNK was increased upon the treatment with erastin and this induced phosphorylation was further enhanced by sodium chlorate (Figure $7 \mathrm{a}-\mathrm{c}$ ). In contrast, the erastin-induced increase in HO-1 expression was not changed significantly by treatment with sodium chlorate (Figure $7 \mathrm{a}, \mathrm{d}$ ). These results suggest that the activation of the JNK pathway is involved in reduced-sulfation enhanced oxidative stress, whereas the Nrf2-ARE pathway is not influenced by reduced sulfation in the cells. 
(a)

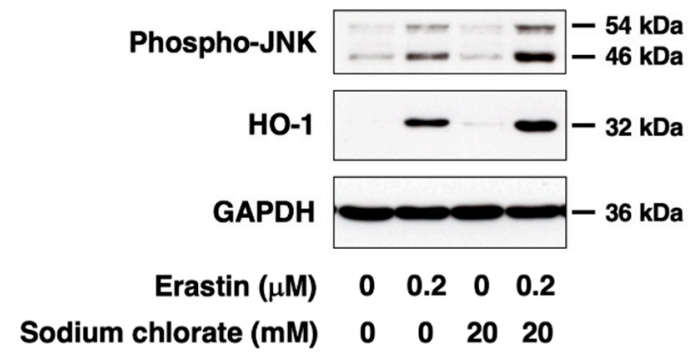

(b)

Phospho-JNK (46 kDa)

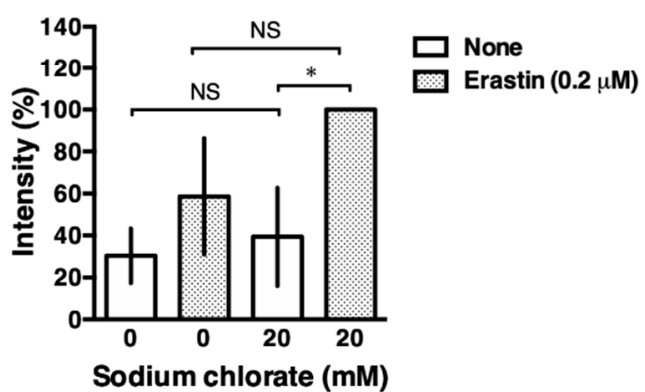

(c)

Phospho-JNK (54 kDa)

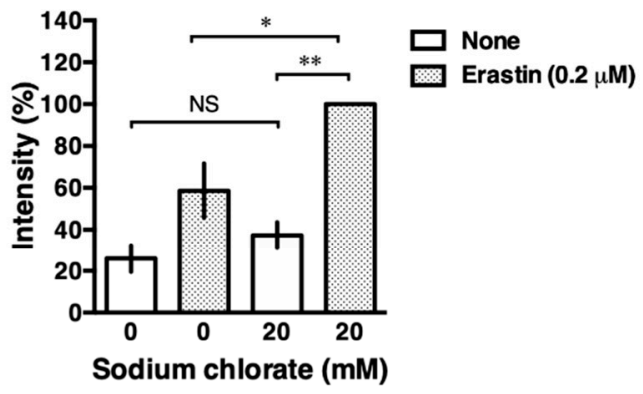

(d)

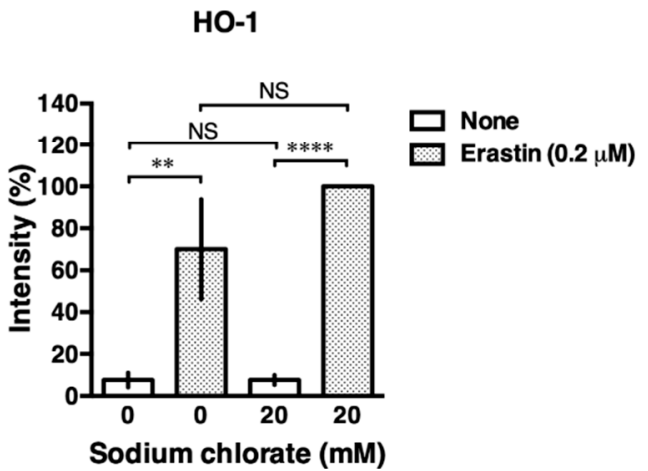

Figure 7. Reduced sulfation enhanced phosphorylation of JNK. (a) Western blot analysis of phospho-JNK and HO-1. (b-d) The band intensity was quantified using an Image J software. The data are presented as the mean $\pm \mathrm{SD}$. The data were obtained from at least three independent experiments. ${ }^{*} p<0.05$, ** $p<0.01,{ }^{* * * *} p<0.0001$; NS, not significant.

\section{Discussion}

In this study, we examined the role of sulfation in oxidative stress using mouse hippocampal HT22 cells, a well-established cellular model for studying endogenous oxidative stress [4]. We found that reduced sulfation exacerbated both glutamate-induced oxytosis and erastin-induced ferroptosis via enhancing ROS production and $\mathrm{Ca}^{2+}$ influx. Our results suggest that the reduced sulfation influences the progression of oxidative stress-induced cell death, which occurs in a variety of neurodegenerative diseases.

Sodium chlorate appeared to be the most effective of the substance used in reducing the sulfation of a variety of macromolecules [26]. On the other hand, sodium chlorate, a herbicide and major water disinfectant byproduct, generates reactive oxygen species and induces oxidative damage in human erythrocytes [27]. Treatment of HT22 cells with sodium chlorate alone did not induce ROS production (Figure 5b) and did not affect cellular antioxidant system such as GSH content (Figure 5a). Ali et al. proposed that chlorate enters the erythrocyte, probably through band 3 anion channel, where it is reduced to chloride ion and this process generates ROS and reactive nitrogen species [27]. However, at present, it is uncertain whether band 3 anion channel can function in HT22 cells. Because chlorate is an in vitro inhibitor of ATP-sulfurylase, the first enzyme in the biosynthesis of 3 '-phosphoadenosine $5^{\prime}$-phosphosulfate which is the ubiquitous co-substrate for sulfation [28], the exact mediators of ROS generation are not clear.

Previous studies have suggested that GAGs play an important role in the pathogenesis and alleviation of neurodegenerative disorders, including Alzheimer's disease and Parkinson's disease. In vitro studies showed that CSPGs protected excitotoxic cell death induced by glutamate in hippocampal and cortical primary neurons and suggested that CSPGs exert their neuroprotective action by antagonizing cellular responses following the activation of excitatory amino acid receptors rather than blocking the activation of certain receptor subtypes $[29,30]$. Whether this neuroprotection is 
dependent upon sulfation of the GAG chains is not clear. In this study, use of $\beta$-D-xyloside, an inhibitor of GAG chain synthesis [20], proved that reduced GAG chains on HSPGs and CSPGs influenced endogenous oxidative stress-induced cell death similarly to sodium chlorate. Other studies showed that HS had a significant neuroprotective effect in the face of ibotenic acid brain injury [31]. The buffering features of CSPGs are supported by their role on determining the local diffusion properties of calcium in the brain extracellular space [32]. This mechanism is also suggested to be involved in neuroprotection against iron-induced cell death by perineuronal nets, a specialized form of ECM which mainly consist of CSPGs [13]. Collectively, negative charges on HSPGs and CSPGs may influence the diffusion of divalent cations such as $\mathrm{Ca}^{2+}$ and $\mathrm{Fe}^{2+}$ which play a critical role in glutamate excitotoxicity. Here, we demonstrated that sodium chlorate weakened the protective effect of an iron chelator, deferoxamine, on erastin-induced cell death. Both $\mathrm{Ca}^{2+}$ and $\mathrm{Fe}^{2+}$ are involved intimately in glutamate- and erastin-induced cell death in HT22 cells [33], suggesting that reduced sulfation by the treatment with sodium chlorate enhances diffusion of divalent cations and exacerbates glutamate- and erastin-induced oxidative stress.

Western blot analysis revealed that sodium chlorate enhanced erastin-induced phosphorylation of JNK. Because it has been well established that ROS are potent inducers of JNK, it is probable that sodium chlorate enhanced JNK phosphorylation by increasing ROS production [34]. In addition, reduced sulfation may affect JNK phosphorylation extracellularly. It has been reported that the subendothelial ECM modulates JNK activation by flow [35]. These findings suggest that JNK activity could be modulated extracellularly by not only fluid shear stress, but also reduced sulfation. Taken together, our results support a role for sulfation in the maintenance of cell survival and prevention of oxidative-stress induced cell death.

\section{Conclusions}

We investigated the role of sulfation on endogenous oxidative stress such as glutamate-induced oxytosis and erastin-induced ferroptosis using mouse hippocampal HT22 cells. The widely used sulfation inhibitor sodium chlorate and $\beta$-D-xyloside, which prevents proteoglycan glycosaminoglycan chain attachment, both reduced HS and CS, and exacerbated glutamate- and erastin-induced cell death, suggesting that extracellular matrix influenced oxytosis and ferroptosis. Moreover, sodium chlorate enhanced the generation of ROS and influx of extracellular $\mathrm{Ca}^{2+}$ in the process of oxytosis and ferroptosis. Western blot analysis revealed that sodium chlorate enhanced erastin-induced JNK phosphorylation, which is preferentially activated cell stress-inducing signals. Our findings indicate that sulfation is an important modification for neuroprotection against oxytosis and ferroptosis in neuronal hippocampal cells.

Author Contributions: All authors have read and agree to the published version of the manuscript. Conceptualization, Y.H. and Y.K.; validation, H.N. and K.O.-h.; formal analysis, K.O.-h.; investigation, H.N.; writing—original draft preparation, H.N.; Y.K.; H.M.G.; Y.H.; visualization, H.N.; supervision, Y.H. and Y.K.; project administration, Y.H, and H.M.G.; funding acquisition, Y.H. All authors have read and agreed to the published version of the manuscript.

Funding: This research received no external funding.

Acknowledgments: We thank Hiromitsu Murai for contributing to the initial experiments. We are very grateful to David Schubert for his generous gift of HT22 cells. H.N. is supported by TOBITATE! (Leap for tomorrow) of Ministry of Education, Culture, Sports, Science and Technology, Japan.

Conflicts of Interest: The authors declare no conflict of interest.

\section{Abbreviations}

CS, chondroitin sulfate; CSPGs, chondroitin sulfate proteoglycans; DMEM, Dulbecco's modified Eagle's medium; ECM, extracellular matrix; FITC, fluorescein isothiocyanate; GAGs, glycosaminoglycans; GSH, glutathione; HO-1, hemoxygenase-1; HS, heparan sulfate; HSPGs, heparan sulfate proteoglycans; JNK, c-Jun N-terminal kinase; LDH, lactate dehydrogenase; PBS, phosphate-buffered saline; ROS, reactive oxygen species; SDS, sodium dodecyl sulfate. 


\section{References}

1. Lewerenz, J.; Ates, G.; Methner, A.; Conrad, M.; Maher, P. Oxytosis/Ferroptosis-(Re-) emerging roles for oxidative stress-dependent non-apoptotic cell death in diseases of the central nervous system. Front. Neurosci. 2018, 12, 214. [CrossRef] [PubMed]

2. Maher, P.; Davis, J.B. The role of monoamine metabolism in oxidative glutamate toxicity. J. Neurosci. 1996, 16, 6394-6401. [CrossRef] [PubMed]

3. Stockwell, B.R.; Friedmann Angeli, J.P.; Bayir, H.; Bush, A.I.; Conrad, M.; Dixon, S.J.; Fulda, S.; Gascon, S.; Hatzios, S.K.; Kagan, V.E.; et al. Ferroptosis: A regulated cell death nexus linking metabolism, redox biology, and disease. Cell 2017, 171, 273-285. [CrossRef] [PubMed]

4. Tan, S.; Schubert, D.; Maher, P. Oxytosis: A novel form of programmed cell death. Curr. Top. Med. Chem. 2001, 1, 497-506.

5. Murphy, T.H.; Miyamoto, M.; Sastre, A.; Schnaar, R.L.; Coyle, J.T. Glutamate toxicity in a neuronal cell line involves inhibition of cystine transport leading to oxidative stress. Neuron 1989, 2, 1547-1558. [CrossRef]

6. Ishige, K.; Schubert, D.; Sagara, Y. Flavonoids protect neuronal cells from oxidative stress by three distinct mechanisms. Free Radic. Biol. Med. 2001, 30, 433-446. [CrossRef]

7. Aoun, P.; Watson, D.G.; Simpkins, J.W. Neuroprotective effects of PPARgamma agonists against oxidative insults in HT-22 cells. Eur. J. Pharm. 2003, 472, 65-71. [CrossRef]

8. Hirata, Y.; Yamada, C.; Ito, Y.; Yamamoto, S.; Nagase, H.; Oh-Hashi, K.; Kiuchi, K.; Suzuki, H.; Sawada, M.; Furuta, K. Novel oxindole derivatives prevent oxidative stress-induced cell death in mouse hippocampal HT22cells. Neuropharmacology 2018, 135, 242-252. [CrossRef] [PubMed]

9. Campo, G.M.; Avenoso, A.; Campo, S.; Ferlazzo, A.M.; Calatroni, A. Antioxidant activity of chondroitin sulfate. Adv. Pharm. 2006, 53, 417-431.

10. Lucena, S.V.; Moura, G.; Rodrigues, T.; Watashi, C.M.; Melo, F.H.; Icimoto, M.Y.; Viana, G.M.; Nader, H.B.; Monteiro, H.P.; Tersariol, I.L.S.; et al. Heparan sulfate proteoglycan deficiency up-regulates the intracellular production of nitric oxide in Chinese hamster ovary cell lines. J. Cell. Physiol. 2018, 233, 3176-3194. [CrossRef]

11. Campo, G.M.; D’Ascola, A.; Avenoso, A.; Campo, S.; Ferlazzo, A.M.; Micali, C.; Zanghi, L.; Calatroni, A. Glycosaminoglycans reduce oxidative damage induced by copper $(\mathrm{Cu}+2)$, iron $(\mathrm{Fe}+2)$ and hydrogen peroxide $\left(\mathrm{H}_{2} \mathrm{O}_{2}\right)$ in human fibroblast cultures. Glycoconj. J. 2004, 20, 133-141. [CrossRef] [PubMed]

12. Dudas, B.; Rose, M.; Cornelli, U.; Pavlovich, A.; Hanin, I. Neuroprotective properties of glycosaminoglycans: Potential treatment for neurodegenerative disorders. Neurodegener. Dis. 2008, 5, 200-205. [CrossRef] [PubMed]

13. Suttkus, A.; Rohn, S.; Jager, C.; Arendt, T.; Morawski, M. Neuroprotection against iron-induced cell death by perineuronal nets-An in vivo analysis of oxidative stress. Am. J. Neurodegener. Dis. 2012, 1, 122-129. [PubMed]

14. Fadel, S.; Eley, A. Chlorate: A reversible inhibitor of proteoglycan sulphation in Chlamydia trachomatis-infected cells. J. Med. Microbiol. 2004, 53, 93-95. [CrossRef] [PubMed]

15. Humphries, D.E.; Silbert, J.E. Chlorate: A reversible inhibitor of proteoglycan sulfation. Biochem. Biophys. Res. Commun. 1988, 154, 365-371. [CrossRef]

16. Galligani, L.; Hopwood, J.; Schwartz, N.B.; Dorfman, A. Stimulation of synthesis of free chondroitin sulfate chains by $\beta$-D-xylosides in cultured cells. J. Biol. Chem. 1975, 250, 5400-5406.

17. Schwartz, N.B.; Domowicz, M.S. Proteoglycans in brain development and pathogenesis. FEBS Lett. 2018, 592, 3791-3805. [CrossRef]

18. Hissin, P.J.; Hilf, R. A fluorometric method for determination of oxidized and reduced glutathione in tissues. Anal. Biochem. 1976, 74, 214-226. [CrossRef]

19. van den Born, J.; Salmivirta, K.; Henttinen, T.; Ostman, N.; Ishimaru, T.; Miyaura, S.; Yoshida, K.; Salmivirta, M. Novel heparan sulfate structures revealed by monoclonal antibodies. J. Biol. Chem. 2005, 280, 20516-20523. [CrossRef]

20. Carrino, D.A.; Caplan, A.I. The effects of $\beta$-D-xyloside on the synthesis of proteoglycans by skeletal muscle: Lack of effect on decorin and differential polymerization of core protein-bound and xyloside-linked chondroitin sulfate. Matrix Biol. 1994, 14, 121-133. [CrossRef]

21. Behl, C.; Widmann, M.; Trapp, T.; Holsboer, F. 17- $\beta$ estradiol protects neurons from oxidative stress-induced cell death in vitro. Biochem. Biophys. Res. Commun. 1995, 216, 473-482. [CrossRef] [PubMed] 
22. Jelinek, A.; Heyder, L.; Daude, M.; Plessner, M.; Krippner, S.; Grosse, R.; Diederich, W.E.; Culmsee, C. Mitochondrial rescue prevents glutathione peroxidase-dependent ferroptosis. Free Radic. Biol. Med. 2018, 117, 45-57. [CrossRef] [PubMed]

23. Hirata, Y.; Ito, Y.; Takashima, M.; Yagyu, K.; Oh-Hashi, K.; Suzuki, H.; Ono, K.; Furuta, K.; Sawada, M. Novel oxindole-curcumin hybrid compound for antioxidative stress and neuroprotection. ACS Chem. Neurosci. 2020, 11, 76-85. [CrossRef] [PubMed]

24. Johnson, J.A.; Johnson, D.A.; Kraft, A.D.; Calkins, M.J.; Jakel, R.J.; Vargas, M.R.; Chen, P.C. The Nrf2-ARE pathway: An indicator and modulator of oxidative stress in neurodegeneration. Ann. N. Y. Acad. Sci. 2008, 1147, 61-69. [CrossRef]

25. Hirata, Y.; Iwasaki, T.; Makimura, Y.; Okajima, S.; Oh-Hashi, K.; Takemori, H. Inhibition of double-stranded RNA-dependent protein kinase prevents oxytosis and ferroptosis in mouse hippocampal HT22 cells. Toxicology 2019, 418, 1-10. [CrossRef] [PubMed]

26. Safaiyan, F.; Kolset, S.O.; Prydz, K.; Gottfridsson, E.; Lindahl, U.; Salmivirta, M. Selective effects of sodium chlorate treatment on the sulfation of heparan sulfate. J. Biol. Chem. 1999, 274, 36267-36273. [CrossRef]

27. Ali, S.N.; Ahmad, M.K.; Mahmood, R. Sodium chlorate, a herbicide and major water disinfectant byproduct, generates reactive oxygen species and induces oxidative damage in human erythrocytes. Env. Sci. Pollut. Res. Int. 2017, 24, 1898-1909. [CrossRef]

28. Baeuerle, P.A.; Huttner, W.B. Chlorate-A potent inhibitor of protein sulfation in intact cells. Biochem. Biophys. Res. Commun. 1986, 141, 870-877. [CrossRef]

29. Okamoto, M.; Mori, S.; Endo, H. A protective action of chondroitin sulfate proteoglycans against neuronal cell death induced by glutamate. Brain Res. 1994, 637, 57-67. [CrossRef]

30. Okamoto, M.; Mori, S.; Ichimura, M.; Endo, H. Chondroitin sulfate proteoglycans protect cultured rat's cortical and hippocampal neurons from delayed cell death induced by excitatory amino acids. Neurosci. Lett. 1994, 172, 51-54. [CrossRef]

31. Naylor, M.C.; Negia, M.; Noetzel, M.; Burns, T.C.; Demorest, Z.L.; Low, W.C. Heparan sulfate mediates neuroprotection from degeneration in experimental glutaric aciduria. Cell Transpl. 2007, 16, 187-195. [CrossRef] [PubMed]

32. Hrabetova, S.; Masri, D.; Tao, L.; Xiao, F.; Nicholson, C. Calcium diffusion enhanced after cleavage of negatively charged components of brain extracellular matrix by chondroitinase ABC. J. Physiol. 2009, 587, 4029-4049. [CrossRef] [PubMed]

33. Takashima, M.; Ichihara, K.; Hirata, Y. Neuroprotective effects of Brazilian green propolis on oxytosis/ferroptosis in mouse hippocampal HT22 cells. Food Chem. Toxicol. 2019, 132, 110669. [CrossRef] [PubMed]

34. Shen, H.M.; Liu, Z.G. JNK signaling pathway is a key modulator in cell death mediated by reactive oxygen and nitrogen species. Free Radic. Biol. Med. 2006, 40, 928-939. [CrossRef]

35. Hahn, C.; Orr, A.W.; Sanders, J.M.; Jhaveri, K.A.; Schwartz, M.A. The subendothelial extracellular matrix modulates JNK activation by flow. Circ. Res. 2009, 104, 995-1003. [CrossRef]

(C) 2020 by the authors. Licensee MDPI, Basel, Switzerland. This article is an open access article distributed under the terms and conditions of the Creative Commons Attribution (CC BY) license (http://creativecommons.org/licenses/by/4.0/). 